\title{
Contributo para a compreensão do financiamento da saúde em Portugal: $O$ caso dos cuidados de saúde primários, dos cuidados hospitalares e dos cuidados continuados.
}

\section{Contribution to the understanding of the health financingin Portugal: The case of primary healthcare, hospitals and long-term care.}

\section{Contribución a la comprensión de la financiación de la salud en Portugal: El caso de la atención primaria de salud, la atención hospitalaria y de atención a largo plazo.}

RESUMO: O sistema de saúde português assenta num mix de fontes de financiamento público e privado, tal como acontece em vários países europeus.Dada a sua complexidade, pareceu útil desenvolver um artigo com o objetivo de contribuir para a compreensão do financiamento de saúde em Portugal, tendo em conta as particularidades dos cuidados de saúde primários, dos cuidados hospitalares e ainda dos cuidados continuados.Para esse efeito, foi realizada uma revisão de literatura, com recurso à B-on (Biblioteca do Conhecimento OnlineB-ON - Biblioteca do conhecimento online), através da opção "pesquisa global”, utilizando os descritores financiamento, saúde, Portugal. Como critérios de inclusão foram considerados a existência de um resumo, a disponibilidade integral do artigo online, o período temporal de publicação entre 2005-2015 e o facto de respeitar em concreto o setor da saúde. Os resultados obtidos permitiram a descrição e explicitação

\footnotetext{
1 Escola Nacional de Saúde Pública, Universidade Nova de Lisboa. Portugal.E-mail:anaescoval@ensp.unl.pt 2 Associação Portuguesa para o Desenvolvimento Hospitalar (APDH).E-mail:a.livio@apdh.pt; a.livio@ensp.unl.pt 3 Escola Nacional de Saúde Pública, Universidade Nova de Lisboa. Portugal. E-mail: patbarbosa@ensp.unl.pt
} 
das formas de financiamento utilizados no setor da saúde em Portugal, assim como apresentar o modelo de financiamento nos vários níveis de cuidados: primários, hospitalares e continuados. Palavras-chave: sistema de saúde; financiamento; saúde; Portugal.

ABSTRACT: The Portuguese health system is based on a mix of sources of public and private funding, as is the case in many European countries. Given its complexity, it seemed useful to develop an article with the purpose of contributing to the understanding of health financing in Portugal, taking into account the particularities of primary health care, hospital and long-term care. To this end, a literature review was conducted, using the B-on (Biblioteca do conhecimento onlineOnline Knowledge Library), through the "global search" option, using the descriptorsfinancing, health, Portugal. The inclusion criteria considered were the existence of a summary, the online availability of full text of article, the time period of the publication between the years 20052015 and the fact that concerns in particular the health sector. The results allowed the description and explanation of the approaches of financing used in the health sector in Portugal, as well as present the financing model in the several levels of care: primary, hospital and long-term. Keywords: health system; financing; health; Portugal.

RESUMEN: El sistema de salud portugués se basa en una mezcla de fuentes de financiación pública y privada, como sucede en muchos países europeos. Dada su complejidad, se ha considerado conveniente desarrollar un artículo con el objeto de contribuir para la comprensión de la financiación de la salud en Portugal, teniendo en cuenta las particularidades de la atención primaria de salud, atención hospitalaria e incluso el cuidado a largo plazo. Con este fin, se realizó una revisión de la literatura, mediante el b-on (Biblioteca do Conhecimento Online), a través de la opción "búsqueda global", utilizando los siguientes descriptores: financiación, salud, Portugal. Como criterios de inclusión se consideraron, la existencia de un resumen, la integra disponibilidad del artículo online, el período temporal de la publicación entre 2005-2015 y el hecho de que respecte, en particular, el sector de la salud. Los resultados permitieron la descripción y explicación de las formas de financiación utilizadas en el sector de la salud en Portugal, así como presentar el modelo de financiación en los distintos niveles de atención: primaria, hospitalaria y a largo plazo. Palabras clave: sistema de salud; financiación; salud; Portugal.

\section{INTRODUÇÃO}

O sistema de saúde português, à semelhança de muitos dos sistemas de saúde europeus, assenta num mix de fontes de financiamento público e privado $^{1}$, coexistindo em simultâneo três sistemas distintos: o serviço nacional de saúde (SNS), os subsistemas de saúde (regimes de seguro social de saúde para grupos profissionais específicos, e.g. funcionários públicos, bancários e forças de segurança) e os seguros de saúde voluntários privados. De referir ainda os pagamentos diretos pelos cidadãos.O SNS criado em 1979 é de cobertura universale geral e, tendo em conta as condições económicas e sociais dos cidadãos, tendencialmente gratuito ${ }^{2}$. Em termos de financiamento, o 
SNS é predominantemente financiado pelos impostos (tributação geral), todavia, os copagamentos, designados em algumas circunstâncias por taxas moderadoras, têm vindo a aumentar, sobretudo nos últimos anos, sendo considerados embora sem peso significativo para o financiamento da saúde.

Figura 1- Evolução das taxas moderadoras em Portugal, 2003-2013

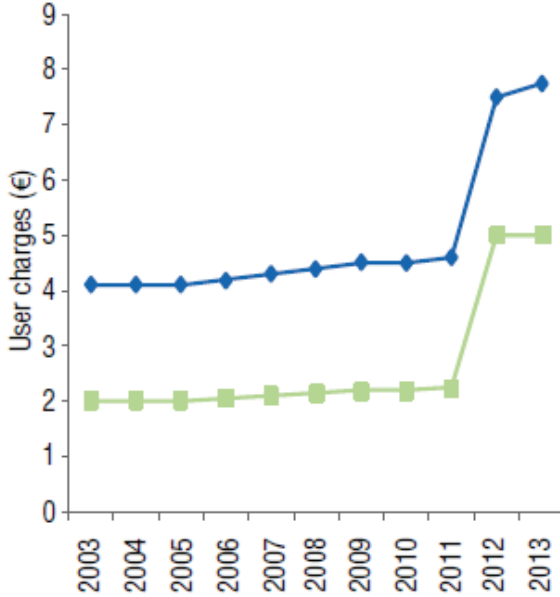

$\leadsto$ Hospital

(specialist consultations)

- $\mathrm{PHC}$ consultations

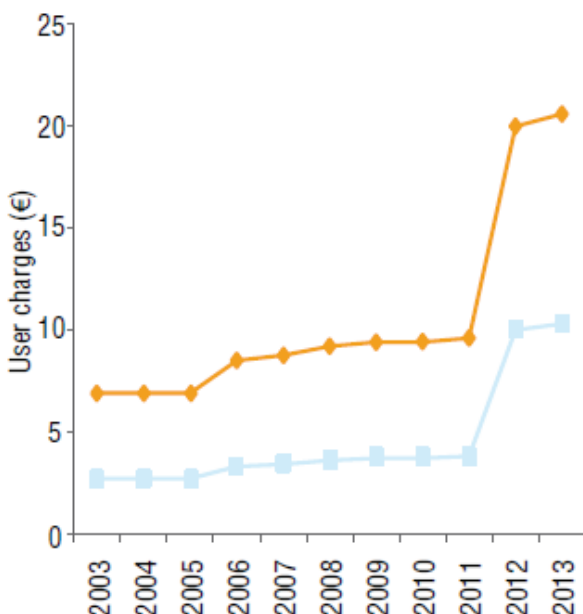

-Hospital
basic emergency

PHC emergency

Note: PHC: public health centre.

Source: ACSS, 2012b.

Fonte: Sakellarides C,et. al. Case study.The impact of the financial crisis on the health system and health in Portugal. European Observatory on Health Systems and Policies. Denmark: World Health Organization; 2014.

Em termos estruturais, o Serviço Nacional de Saúde (SNS), de acordo com dados publicados em 2012, tinha instalada uma rede hospitalar que integrava 214 hospitais (104 hospitais privados e 110 hospitais tutelados pelo Estado, dos quais 103 eram hospitais de acesso universal e 7 hospitais militares ou prisionais). ${ }^{3}$ Ao nível dos cuidados de saúde primários (CSP), estavam previstos 70 Agrupamentos de Centros de Saúde (ACES), e existiam já 186 Unidades de Saúde Familiar (contabilizando 200 em 2013), 167 (em 2013 passaram a 163) Unidades de Cuidados de Saúde Primários e 84 Unidades de Cuidados na Comunidade (88 em 2013), além das outras unidades funcionais: unidades de saúde pública e unidades de recursos assistenciais partilhados (uma por ACES). ${ }^{4}$

O sistema de financiamento do SNS processa-se através do Ministério das Finanças, que anualmente define um orçamento com base no histórico da despesa e nos planos apresentados pelo Ministério da Saúde (MS). Numa fase final, o MS recebe um orçamento global para o SNS, com origem no orçamento Geral do Estado, o qual é afetado às instituições que integram o SNS, sob a gestão dos seus organismos centrais e periféricos (integrados na administração indirecta do Estado) (ver figura 2. Infra). 
Figura 2 Sistema de saúde português: fluxos financeiros

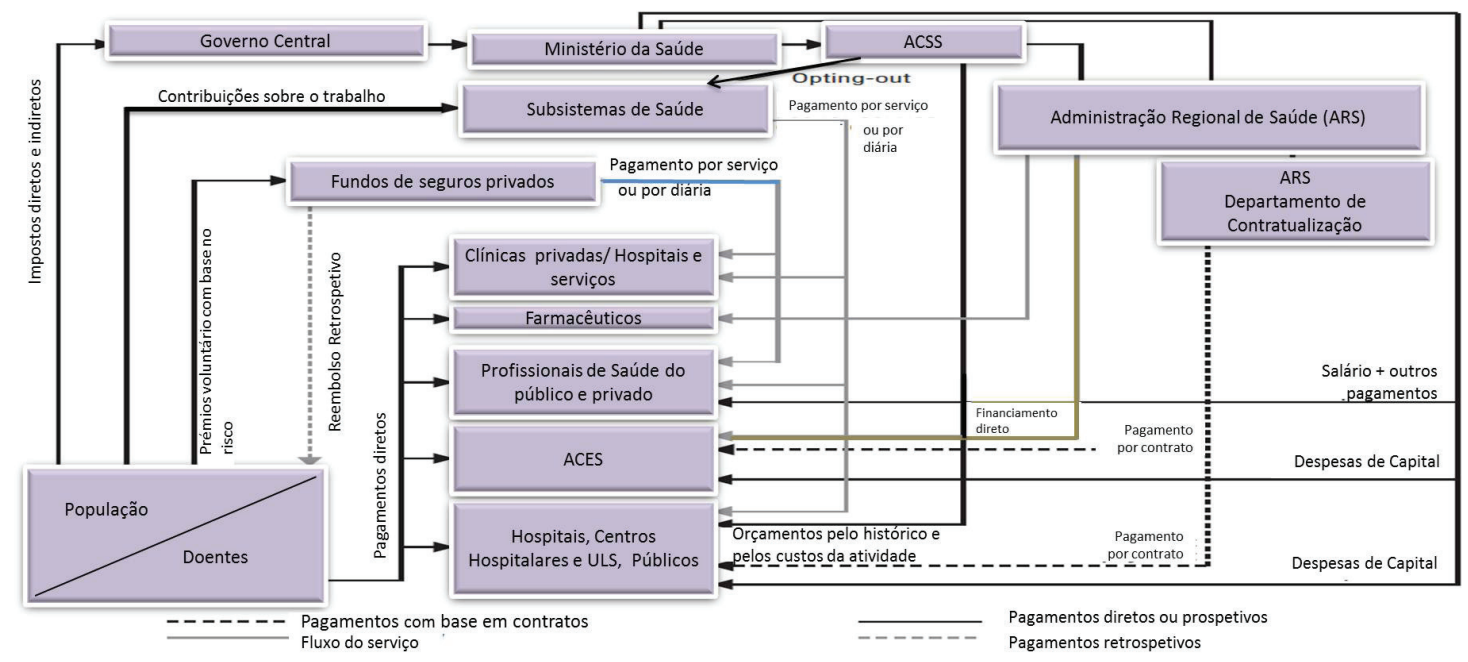

Fonte: Adaptado de Barros P, Machado S, Simões, J. Portugal.Health system review. Health Systems in Transition. 2011; 13(4):1-156.

A conceção do modelo de financiamento do SNS é da responsabilidade da Administração Central do Sistema de Saúde (ACSS), no qual é definida a distribuição dos recursos financeiros por cada região de saúde, com base nas características da população e de acordo com um modelo de capitação ajustada (introduzido em 2012), sob a responsabilidade de cada Administração Regional de Saúde (ARS) - Norte, Centro, Lisboa e Vale do Tejo, Alentejo e Algarve. Compete assim às ARS efetivar o pagamento destas verbas aos diferentes níveis de cuidados, dos quais, considerando a totalidade do orçamento afeto ao SNS, 36,7\% são afetos à prestação de cuidados de saúde primários (CSP), 50,8\% são afetos à prestação de cuidados diferenciados, também designados secundários (hospitais, centros hospitalares e unidades locais de saúde EPE, Hospitais SPA e Hospitais PPP) e $1,8 \%$ é afeto aos cuidados continuados e integrados (dados de 2013). ${ }^{1,5,6,7}$

Acresce referir que Portugal desde a década de noventa que iniciou o desenvolvimento do processo de contratualização, enquanto importante instrumento de apoio ao financiamento numa perspetiva de maior equidade e garantia de acesso aos cidadãos. A contratualização tem por base o relacionamento entre financiadores, compradores e prestadores, assente numa filosofia contratual, na qual são estabelecidos os resultados em saúde esperados para os níveis de financiamento disponibilizados. ${ }^{8,9}$ Isto é, através da assinatura de um Contrato-Programa (CP) são definidos os objetivos institucionais a atingir, que reflectem metas de produção, de desempenho e de qualidade, tendo como contrapartida um determinado nível de financiamento e pressupondo assegurada a autonomia e responsabilização de todas as partes, tendo por base um sistema de incentivos institucionais. O CP, com vista ao acompanhamento e monitorização das instituições, define ainda um conjunto de indicadores que permitem aferir o nível de desempenho destas na perspectiva de garantia dos princípios de acesso e qualidade, desempenho económico-financeiro e assistencial.9,10

4 EPE - Entidade Pública Empresarial; PPP- Parceria Público Privada; SPA - Sector Público Administrativo 


\section{$21 / /$}

Este processo envolve vários intervenientes, designadamente o MS, a ACSS, as ARS e as entidades prestadoras de cuidados (Hospitais, Centros Hospitalares, Unidades Locais de Saúde e os Agrupamentos de Centros de Saúde). ${ }^{9}$

Os CSP constituem-se, idealmente, como a "porta de entrada" no SNS. Os Agrupamentos de Centros de Saúde (ACES), serviços públicos desconcentrados das ARS, com autonomia administrativa, são constituídos por várias unidades funcionais (UF) (nomeadamente, unidades de saúde familiar e unidades de cuidados de saúde personalizados, unidades de cuidados na comunidade, unidades de saúde pública e unidades de recursos assistenciais partilhados), e têm por missão garantir a prestação de cuidados de saúde à população. ${ }^{11,12}$

O processo de contratualização (contratação de objetivos com base nas necessidades de saúde e nos recursos disponíveis) com os CSP é centrado nas necessidades em saúde das diferentes populações, tendo por base a contratação de cuidados de saúde, estando organizado em dois subprocessos: (a) a contratualização externa realizada entre as ARS e os ACES, formalizada pela negociação de Planos de Desempenho e a assinatura de Contrato-Programa e (b) a contratualização interna, realizada entre os ACES e as UF, formalizada pela assinatura das cartas de compromisso. ${ }^{12}$

A contratualização externa entre as ARS e os ACES assenta numa matriz de 20 indicadores de Desempenho divididos em três eixos - nacional (composto por 14 indicadores ao nível do desempenho assistencial, económico e da satisfação), regional (composto por 4 indicadores) e local (composto por 2 indicadores). A contratualização interna entre os ACES e as unidades de saúde familiar (USF) e mais recentemente também com as unidades de cuidados de saúde personalizados (UCSP), pressupõe uma matriz de 22 indicadores com vista à atribuição de incentivos institucionais às USF:

- 12 são de âmbito nacional (ao nível do acesso, desempenho assistencial e económico e satisfação), sendo definidos pelas ARS de acordo com as prioridades das políticas de saúde, comuns a todas as USF e UCSP (revistos a cada 3 anos);

- 4 indicadores são de âmbito regional (ao nível do acesso, desempenho assistencial), sendo definidos da mesma forma que os 12 indicadores a nível nacional;

- 2 indicadores são de âmbito local (ao nível do acesso, desempenho assistencial), definidos, a cada 3 anos, pelos ACES específicos para as suas USF e UCSP;

- 4 indicadores são de âmbito local (ao nível do acesso, desempenho assistencial), mas propostos pelas USF e UCSP (triénio). ${ }^{12}$

De referir ainda que as USF se dividem atualmente em dois modelos distintos (A e B), sendo o Modelo A um modelo em fase de aprendizagem e aperfeiçoamento do trabalho em equipa e 
um primeiro passo para a contratualização interna e o Modelo B caracterizado por um maior amadurecimento organizacional, cujo trabalho em equipa deve refletir a prática efetiva, bem como a aceitação pelos profissionais de níveis de desempenho mais exigentes. Neste modelo são atribuídos incentivos institucionais (com base nos princípios do modelo A) e financeiros aos profissionais aos médicos são atribuídos incentivos aferindo atividades específicas; aos enfermeiros e assistentes técnicos, os incentivos são atribuídos em função dos resultados obtidos pela equipa, como parte da remuneração prevista para os profissionais das USF. ${ }^{12}$

Ao nível dos cuidados secundários, de entre os hospitais públicos, os hospitais SPA representam estabelecimentos públicos, dotados de personalidade jurídica, autonomia administrativa e financeira, com ou sem autonomia patrimonial e os hospitais EPE, representam uma pessoa coletiva de direito público de natureza empresarial dotada de autonomia administrativa, financeira e patrimonial. Estes são financiados através de orçamentos globais baseados em contratos trianuais assinados com o MS. Trata-se de um modelo de pagamento prospetivo, baseado na classificação de doentes Grupos Diagnósticos Homogéneos (GDH), que assenta na atividade realizada, compreendendo o agrupamento sistemático dos doentes em Grupos de Diagnóstico Homogéneos (GDH) ${ }^{5}$ agrupador de GDH All Patient Refined DRG 30 -APR30) e a atribuição de orçamentos ajustados pelo casemix do hospital..$^{1,5,13}$

Os GDH são um sistema de classificação de doentes agudos internados, que permite definir, operacionalmente, os produtos de um hospital. ${ }^{14} \mathrm{O}$ índice de casemix (ICM) de um hospital resulta do rácio entre o número de doentes equivalentes ponderados pelos pesos relativos dos respetivos GDH e o número total de doentes equivalentes. ${ }^{7,15}$

Quanto ao processo de contratualização está igualmente organizado em dois subprocessos: a contratualização externa e interna. O processo de contratualização interna deve ter como princípio a valorização da governação clínica, o desempenho assistencial e a sustentabilidade económicofinanceira dos serviços e das instituições, assegurando que «os compromissos e os objetivos são assumidos internamente (...) e desagregados pelos diferentes (...) serviços segundo uma filosofia de prestação de contas a todos os níveis (...)». ${ }^{7}$ Tal deve ser feito através de um modelo de gestão participada e de proximidade. ${ }^{7}$

Relativamente à metodologia de contratualização externa dos serviços de saúde entre as instituições hospitalares e o MS, após publicação, por despacho do secretário de Estado da Saúde,

5 Os GDH constituem um sistema de classificação de doentes internados em hospitais de agudos que agrupa doentes em grupos clinicamente coerentes e similares do ponto de vista do consumo de recursos. Cada grupo é associado um peso relativo, ou seja, o peso relativo de cada GDH está diretamente relacionado com o nível de severidade atribuído, o nível de severidade tem impacto direto no valor de ICM obtido levando a que quanto menos severa for a produção (ou seja, quanto mais a produção esteja concentrada nos níveis 1 e 2 de severidade), menor seja o ICM.7,13 A metodologia de cálculo dos pesos relativos de cada GDH conjuga duas fontes de informação (a Base de dados central de GDH e Custos de Internamento, de Cuidados Intensivos e das admissões urgentes que deram origem a internamentos), que permitem imputar a cada GDH os custos em função dos dias de internamento por doente em cada GDH, do número total de doentes em cada GDH (convertido em doentes equivalentes) e de critérios de repartição específicos para cada rubrica. ${ }^{7,13}$ 
dos limites máximos a contratar pelas ARS com os hospitais e Unidades locais de Saúde (ULS) EPE, é assumido um compromisso pelas instituições para atingir as metas contratualizadas, em função dos recursos disponíveis e das necessidades da população. Este contrato comtempla três instrumentos fundamentais: (a) contratação da atividade assistencial (permite contratar o volume e o mix de serviços face às necessidades da população da região de saúde e adequando a oferta à procura); (b) modalidade de pagamento (permite sinalizar e alinhar os incentivos com o processo global de prestação de cuidados, contribuindo para um melhor desempenho assistencial e económico-financeiro) e; (c) avaliação do desempenho (permite avaliar em áreas prioritárias a nível nacional, e.g. a adequação do acesso ou o desempenho das instituições, o qual é avaliado através de um conjunto de indicadores de processo, outputs e resultados). ${ }^{7}$

No que concerne aos incentivos institucionais, estes são atribuídos de acordo com o cumprimento das metas previamente negociadas com os Departamentos de Contratualização das ARS, para os indicadores selecionados. No caso da contratualização com os Hospitais/Centros Hospitalares, a repartição dos 5\% do contrato-programa afetos a incentivos tem uma distribuição de $60 \%$ para objetivos institucionais comuns (nacional) e 40\% para objetivos institucionais de cada região. ${ }^{7} \mathrm{Em}$ termos dos indicadores a contratualizar, o processo assenta numa matriz de 16 indicadores, que se dividem por três áreas, a saber Acesso (inclui 5 indicadores, e.g. percentagem de primeiras consultas médicas), Desempenhoassistencial (inclui 7 indicadores, e.g. demora média), Desempenho económico-financeiro (inclui 4 indicadores, e.g. acréscimo de dívida vencida). ${ }^{7}$

Destacam-se ainda, a introdução de algumas inovações, entre outras, no atual modelo de contratualização, como a introdução de um ICM único no internamento, resultante da produção cirúrgica e médica e de um índice de consultas subsequentes por Grupo Hospitalar nas consultas externas, e ainda a inclusão no $\mathrm{CP}$ de vários programas verticais, em que o financiamento do tratamento de algumas patologias se define através de um modelo de pagamento por doente tratado (isto é, baseado na unidade "doente tratado"), e.g o programa de tratamento ambulatório de pessoas a viver com infeção VIH/sida, o programa para doentes com Polineuropatia Amiloidótica Familiar em estádio 1 (Paramiloidose PTPAF1), entre outros. ${ }^{7}$

No caso das Unidades Locais de Saúde (ULS), tendo sido implementada a sua primeira experiência em 1999 com a criação da ULS de Matosinhos, EPE (que integrou o Hospital de Pedro Hispano e o ACES de Matosinhos), representam um modelo de gestão integrada de cuidados de saúde primários, hospitalares e continuados, seguindo a metodologia utilizada em cada um dos níveis de cuidados, com já referido em cima, no caso dos CSP e hospitalares. ${ }^{6,16}$

As ULS possuem o mesmo estatuto que os hospitais EPE. A afetação dos recursos financeiros é baseada na capitação global ajustada pelo risco, sendo consideradas as características e

6 A ULS consiste num modelo que tem como objetivo de melhorar a interligação dos cuidados de saúde primários com os cuidados diferenciados, através de uma prestação e gestão integrada de todos os níveis de cuidados de saúde (Decreto-Lei n. ${ }^{\circ}$ 12/15, de 26 de janeiro, DR 1. ${ }^{a}$ Série, N ${ }^{o}$ 17/2015). 
necessidades de saúde da população, traduzidas num coeficiente de ajustamento global baseado em indicadores da população em risco e da prestação de cuidados de saúde. ${ }^{17}$ Concretamente, a modalidade de pagamento das ULS estabelece que o valor per capita de cada ULS é composto por uma componente dos determinantes em saúde da despesa (40\%) e pelo estado de saúde das populações (60\%). A componente de determinantes da despesa baseia-se na variação da despesa (assente num modelo de regressão linear múltipla, com três variáveis - a despesa de cuidados de saúde primários e hospitalares (variáveis independentes) e a despesa total (variável dependente). ${ }^{18}$

No processo de contratualização com as ULS a repartição dos 10\% do contrato-programa afetos ao cumprimento de objetivos é distribuída por $6 \%$ de objetivos dequalidade dos quais 3\% para CSP, alinhados com a contratualização realizada a nível nacional com os ACES e 3\% para cuidados de saúde secundários, alinhados com a contratualização realizada a nível nacional com os hospitais/centros hospitalares, e considerando objetivos de integração de cuidados ao nível do eixo regional. Os restantes 4\% dizem respeito aos objetivos de eficiência/sustentabilidade financeira, alinhados com a contratualização realizada a nível nacional com os hospitais/centros hospitalares. $^{7}$

Ao nível dos cuidados continuados, está implementada, desde 2006, a Rede Nacional de Cuidados Continuados Integrados (RNCCI), que compreende um modelo assente numa lógica da separação de funções (compra/prestação) mediante a contratualização de serviços e a descentralização das responsabilidades de âmbito territorial. ${ }^{19} \mathrm{O}$ planeamento da compra de serviços foi desenvolvido em dois planos de implementação, num modelo de financiamento partilhado e intersectorial entre o Ministério da Saúde e o Ministério do Trabalho e da Solidariedade Social. ${ }^{19,21}$ Os recursos financeiros afetos à Rede resultam em exclusivo das receitas dos Jogos Sociais, conforme estabelecido na Portaria n. ${ }^{\circ}$ 224/2014 de 4 de novembro, ${ }^{20}$ estando consignado, para o ano de 2015, $50 \%$ desta receita líquida para a ACSS com vista ao financiamento da RNCCI. Destas receitas, $33 \%$ das verbas são ainda destinadas a entidades que prosseguem atribuições nos domínios do planeamento, prevenção e tratamento dos comportamentos aditivos e das dependências e 17\% à Direção-Geral da Saúde para financiamento dos programas especiais, nomeadamente na área do VIH/SIDA (8\%), da saúde mental (1\%), das doenças oncológicas (1\%), da prevenção do tabagismo (1\%), entre outros. ${ }^{20,21}$

\section{CONCLUSÃO}

Considerando o modelo de financiamento vigente em Portugal e a atual situação económicofinanceira, que implicou uma forte racionalização dos recursos e um rigoroso controlo da despesa, com impacto expressivo nas organizações de saúde em geral e consequentemente na população, importa refletir sobre a adequação das políticas de saúde vigentes, especificamente ao nível da distribuição dos recursos financeiros no seio do SNS. 
A importância desta discussão torna-se ainda mais evidente se considerarmos que o SNS e as organizações de saúde enfrentam, a par com as tendências internacionais, desafios associados ao envelhecimento e empobrecimento da população, hoje mais informada e exigente, às dificuldades de acesso, ao desenvolvimento das tecnologias da informação e biomédicas, ao aumento das doenças crónicas e de evolução prolongada e ao elevado ónus que estas representam para o sistema.

Dado que os CSP são a "porta" do sistema de saúde e têm como função referenciar para a tipologia de cuidados apropriada, de acordo com os cuidados que os cidadãos efetivamente necessitam e as crescentes necessidades de cuidados continuados e integrados, decorrentes do aumento das doenças crónicas e do número de idosos em Portugal, parece relevante debater, de acordo com as necessidades das populações (envelhecimento, empobrecimento, mobilidade e proximidade, ganhos em saúde), a adequação da distribuição dos recursos financeiros ao nível dos diferentes tipos de cuidados em cada região de saúde, à luz de um novo paradigma de organização de respostas de proximidade.

Sendo esta realidade incontornável importa assim encontrar um modelo de financiamento mais adequado que permita distribuir de forma mais equilibrada o atual orçamente da saúde, que no caso dos cuidados continuados, é manifestamente desajustado, representando apenas 1.8\% em 2012 (cuja criação da RNCCI, em 2006, reflete a necessidade de respostas a nível nacional).

\section{REFERÊNCIAS BIBLIOGRÁFICAS}

1. European Observatory on health Systems and Policies. Health systems in transition (hit) profile of Portugal[internet].update on user charges updates by Pedro Pita Barros;2013 mar. [consult. 26 de set. 2014]. Disponível em http://hspm.org/countries/portugal25062012/livinghit. aspx?Section $=3.1 \% 20$ Health $\% 20$ expenditure $\&$ Type=Section

2. Lei Constitucional n. ${ }^{\circ} 1 / 89$ de 8 de Julho. Segunda revisão constitucional.

3. Instituto Nacional de Estatística. Dia Mundial da Saúde - 7 de abril.2002-2012 [internet].Lisboa: Instituto Nacional de Estatística; 2014 abril. Disponível em http:// www.ine.pt/xportal/xmain?xpid=INE\&xpgid=ine_destaques\&DESTAQUESdest_ boui=215806720\&DESTAQUESmodo $=2$.

4. Entidade Reguladora da Saúde. Relatório Anual Sobre o Acesso a Cuidados de Saúde nos Estabelecimentos do SNS e Entidades Convencionadas (2013). Lisboa: Entidade Reguladora da Saúde; 2014.

5. Barros P, Machado S, Simões, J. Portugal.Health system review. Health Systems in Transition. 2011; 13(4):1-156. 
6. Ministério da Saúde, Administração Central do Sistema de Saúde. Departamento de Planeamento em Saúde. Unidade Formativa “Contratualização nos Serviços de Saúde”. In Formação- Ação Contratualização nos serviços de saúde. OradoresCláudia Borges (Departamento de Gestão e Financiamento de Prestações de Serviços de Saúde, ACSS);2014.

7. Ministério da Saúde, Administração Central do Sistema de Saúde. Departamento de Planeamento em Saúde. Contrato-Programa 2015. Metodologia para definição de preços e fixação de objetivos. Lisboa:Departamento de Gestão e Financiamento de Prestações de Serviços de Saúde, Administração Central do Sistema de Saúde, Ministério da Saúde;2014.

8. Escoval A, Matos T, Ferreira AS, LourençoA.Contratualização interna vs. contratualização externa. Volume temático Contratualização na saúde. Rev Port Saúde Pública.2010; 9: 161-179.

9. Ministério da Saúde, Administração Central do Sistema de Saúde. Departamento de Planeamento em Saúde. Manual do processo de contratualização - hospitais e uls. Lisboa: Departamento de Gestão e Financiamento de Prestações de Serviços de Saúde, Administração Central do Sistema de Saúde, Ministério da Saúde; 2011.

10. Escoval A. O processo de contratualização na saúde em Portugal (1996-2005). Volume temático Contratualização na saúde. Rev Port Saúde Pública. 2010; 9: 7-24.

11. Decreto-Lei n. ${ }^{\circ}$ 28/2008. Diário da República, 1. ${ }^{\text {a }}$ série, n. ${ }^{\circ}$ 38,22 de Fevereiro de 2008.

12. Ministério da Saúde, Administração Central do Sistema de Saúde. Metodologia da Contratualização para os Cuidados de Saúde Primários para o ano de 2014. [internet] Lisboa: Ministério da Saúde, Administração Central do Sistema de Saúde; 2014 jan [consult. 26 de set. 2014].Disponível em http://www.acss.min-saude.pt/DownloadsePublicações/ CuidadosdeSaúdePrimários/tabid/118/language/pt-PT/Default.aspx.

13. Ministério da Saúde, Administração Central do Sistema de Saúde. Produção nacional. Grupos de Diagnósticos Homogéneos.[internet]. Lisboa:Ministério da Saúde, Administração Central do Sistema de Saúde;s.d.[consult. 26 de set. 2014]. Disponível em http://www.acss.min-saude.pt/\%C3\%81reaseUnidades/ DepartamentoGest $\%$ C3\%A3oeFinanciamentoPrestSa\%C3\%BAde/ SClassifica\%C3\%A7\%C3\%A3oDoentes/GruposdeDiagn\%C3\%B3sticosHomog\%C3\%A9neos/ tabid/460/language/pt-PT/Default.aspx.

14. Urbano J, Bentes M. Definição da Produção do Hospital: Os Grupos de Diagnósticos Homogéneos. Rev Port Saúde Pública.1990; 8 (1):49-60

15. Ministério da Saúde, Administração Central do Sistema de Saúde. Preços do SNS. Cálculo do Doente Equivalente e Índice de Case-Mix.[internet]. Lisboa: Ministério da Saúde, 
Administração Central do Sistema de Saúde;s.d. [consult. 26 de set. 2014]. Disponível em http:// www.acss.min-saude.pt/Publica\%C3\%A7\%C3\%B5es/TabelaseImpressos/Pre\%C3\%A7osdoSNS/ tabid/141/language/pt-PT/Default.aspx.

16. Decreto-Lei n. ${ }^{\circ}$ 12/2015. Diários da República 108/99, $1 .^{\text {a}}-$ A série, de 10 de maio de 1999.

17. Ministério da Saúde, Administração Central do Sistema de Saúde. Modalidade de Pagamento da Unidades Locais de Saúde Contrato-Programa 2010-2012. [internet]. Administração Central do Sistema de Saúde, Ministério da Saúde; s.d. Disponível http://www. acss.min-saude.pt/Portals/0/Modalidade\%20de\%20Pagamento\%20ULS\%202010_12n.pdf.

18. Ministério da Saúde, Administração Central do Sistema de Saúde. Unidades Locais de Saúde: modalidade de pagamento : unidade operacional de financiamento e contratualização. [internet]. Lisboa: Administração Central do Sistema de Saúde. Ministério Saúde; 2009 março. [consult. 26 de set. 2014]. Disponível em http://www.acss.min-saude.pt/Portals/0/ modalidadeULS09.pdf

19. Ministério da Saúde, Unidade de Missão para os Cuidados Continuados Integrados. Relatório de monitorização do desenvolvimento da Rede Nacional de Cuidados Continuados integrados em 2007: Resumo [internet]. Lisboa: Unidade de Missão para os Cuidados Continuados Integrados; 2008. [Consult. em 2 out 2014]. Disponívelemhttp://apfisio.pt/ GrupoTrabalho/RelMonitorizacao.pdf.

20. Portaria n..$^{\circ}$ 224/2014. Diário da República, $1 .^{\text {a }}$ série, n. ${ }^{\circ} 213,4$ de novembro de 2014.

21. Escoval A, Ribeiro R, Matos T, Lívio A. Projeto de Investigação "Contratualização em Cuidados Continuados Integrados. Fase 2: Revisão das práticas internacionais. Lisboa: Universidade Nova de Lisboa, Escola Nacional de Saúde Pública; 2012.

Artigo apresentado em 21-03-15

Artigo aprovado em 06-02-16 Artigo publicado no sistema em 30-03-16 